\title{
Characteristics of Hippocampal Primed Burst Potentiation in vitro and in the Awake Rat
}

\author{
D. M. Diamond, T. V. Dunwiddie, and G. M. Rose \\ Medical Research Service, Veterans Administration Medical Center, and Department of Pharmacology, University of \\ Colorado Health Sciences Center, Denver, Colorado 80220
}

\begin{abstract}
A pattern of electrical stimulation based on 2 prominent physiological features of the hippocampus, complex spike discharge and theta rhythm, was used to induce lasting increases in responses recorded in area CA1 of hippocampal slices maintained in vitro and from the hippocampus of behaving rats. This effect, termed primed burst (PB) potentiation, was elicited by as few as 3 stimuli delivered to the commissural/associational afferents to CA1. The patterns of stimulus presentation consisted of a single priming pulse followed either 140 or $170 \mathrm{msec}$ later by a high-frequency burst of 2-10 pulses; control stimulation composed of unprimed high-frequency trains of up to 10 pulses had no enduring effect. Of all intervals tested, only 140 and 170 msec delays between the priming and burst stimuli were effective. PB potentiation could be induced both homo- and heterosynaptically. In the latter case, the priming pulse and burst stimuli were delivered to different dendritic fields; under these conditions, the PB effect was confined to the "burst" pathway. PB potentiation is not dependent on somal spiking; dendritic activation appears to be both necessary and sufficient for lasting changes to occur. Two findings indicate that PB potentiation and LTP have common mechanisms: (1) The effects of PB stimulation and LTP were not additive, in that saturation of the enhancement by PB stimulation eliminated any further increases in response with LTP stimulation; and (2) both PB potentiation and LTP were prevented if the $\mathrm{N}$-methyl-D-aspartate antagonists 2-amino-5-phosphonovaleric acid or phencyclidine were added to the in vitro perfusion medium. Recordings from the hippocampus of awake rats demonstrated that PB potentiation of the CA1 population spike and slope of the EPSP are reliably induced under physiological conditions. This extensive characterization of PB stimulation provides novel information regarding the physiological and pharmacological basis of a possible role of endogenous rhythms in the processing and storage of information.
\end{abstract}

Several forms of synaptic plasticity that may play a role in processes underlying learning and memory have been described in both the vertebrate and invertebrate nervous systems

Received Nov. 17, 1987; revised Mar. 7, 1988; accepted Apr. 1, 1988.

This work was supported by the Veterans Administration Medical Research Service, NIDA Grant DA02702, and an ADMH Training Grant to D.M.D. We wish to thank Catherine Bennett for helpful criticism of the manuscript.

Correspondence should be addressed to G. M. Rose, Ph.D., Medical Research (151), VAMC, 1055 Clermont St., Denver, CO 80220.

Copyright $\odot 1988$ Society for Neuroscience $0270-6474 / 88 / 114079-10 \$ 02.00 / 0$
(McNaughton, 1983; Lynch and Baudry, 1984; Farley and Alkon, 1985; Byrne, 1987). Of these, a phenomenon termed longterm potentiation (LTP) has received extensive attention because it shares a number of characteristics with memory. For example, LTP has a rapid onset and long duration and is strengthened by repetition (Barnes, 1979; Teyler and Discenna, 1987). In addition, the decay of LTP is correlated with the time course of behaviorally assessed forgetting (Barnes, 1979; Barnes and McNaughton, 1985). The similarity of LTP and memory has fostered an extensive characterization of the conditions underlying the initiation of LTP, as well as its biochemical substrates (Lynch and Baudry, 1984; Wigström and Gustafsson, 1985a, b; Collingridge and Bliss, 1987).

Despite this progress, little attention has been directed toward the issue of how long-term changes in synaptic strength can occur as a function of behavioral learning. Recent findings have led to a partial resolution of this issue. Sharp et al. $(1985,1987)$ showed that exposure of rats to a novel environment results in an LTP-like increase in the effectiveness of electrical stimulation of the hippocampus. Similarly, others have shown that an enhancement of synaptic transmission is correlated with behavioral associative learning (Jaffard and Jeantet, 1981; Weisz et al., 1984). The basis for these changes in responses may be that endogenous patterns of hippocampal neuronal activity that occur during learning can activate mechanisms of memory formation.

Our approach to testing this hypothesis was through the use of electrical stimulation that mimics endogenous patterns of hippocampal activity known to occur in behaving animals. Two such endogenous patterns are complex spike activity, which is composed of brief high-frequency bursts of 2-5 spikes (Ranck, 1973; Suzuki and Smith, 1985) and theta rhythm, a 4-12 Hz cyclic fluctuation of hippocampal unit (Rose, 1983) and slow wave (Vanderwolf, 1969; Buzsaki et al., 1983; Bland, 1986) activity. Previously, we demonstrated that patterned stimulation that mimicked the temporal components of complex spike bursting and theta rhythm resulted in a marked reduction in the threshold for inducing lasting changes in synaptic strength (Rose and Dunwiddie, 1986). The patterned stimulation consisted of a single priming pulse followed $170 \mathrm{msec}$ later (i.e., at a theta frequency of $6 \mathrm{~Hz}$ ) by a high-frequency burst of pulses. This effect, termed primed burst $(\mathrm{PB})$ potentiation, can be reliably evoked in the in vitro CA 1 field by only 5 patterned pulses (Rose and Dunwiddie, 1986). Similar effects involving patterned stimulation and hippocampal synaptic plasticity have been observed in stratum radiatum of CA1 in the awake rat (Staubli and Lynch, 1987) and in vitro (Larson and Lynch, 1986; Larson 
et al., 1986), as well as in the dentate gyrus of anesthetized rats (Greenstein et al., 1988).

In the present study, we have provided an extensive characterization of $\mathrm{PB}$ potentiation. First, we performed parametric analyses of physiological characteristics of $\mathrm{PB}$ potentiation in the CAl cell body layer of the in vitro hippocampal slice. These experiments identified novel features of $\mathrm{PB}$ potentiation, such as its threshold and the necessity of dendritic activation, rather than somal spiking, to elicit lasting effects. Second, pharmacological and physiological manipulations determined that PB potentiation appears to be induced through the same mechanisms as conventionally induced LTP. Finally, we demonstrated that $\mathrm{PB}$ potentiation can be induced in the hippocampus of the awake rat. The findings are discussed in the context of current thcorics of LTP and associative learning and the role of endogenous patterns of hippocampal activity in the generation of learning-induced synaptic strengthening.

\section{Materials and Methods}

\section{In vitro preparation}

The subjects were male Sprague-Dawley albino rats (Harlan Laboratories, Indianapolis, IN) weighing 100-200 gm. Following decapitation, the hippocampi were dissected free, while the brain was chilled at 0 $4^{\circ} \mathrm{C}$ in a medium containing $124 \mathrm{~mm} \mathrm{NaCl}, 4.9 \mathrm{~mm} \mathrm{KCl}, 1.2 \mathrm{~mm}$ $\mathrm{KH}_{2} \mathrm{PO}_{4}, 2.5 \mathrm{~mm} \mathrm{CaCl}_{2}, 2.4 \mathrm{~mm} \mathrm{MgSO}$, $26 \mathrm{~mm} \mathrm{NaHCO}_{3}$, and $10 \mathrm{~mm}$ glucose that had been equilibrated with a $95 \% \mathrm{O}_{2} / 5 \% \mathrm{CO}_{2}$ gas mixture. Transverse slices were cut at $400 \mu \mathrm{m}$ thickness and transferred to a recording chamber (Spencer et al., 1976), where they were kept submerged in the same medium (warmed to $33.5^{\circ} \mathrm{C}$ ) and perfused at a rate of $2 \mathrm{ml} / \mathrm{min}$.

Extracellular recordings of field potentials from the CA1 pyramidal cell layer were made using glass micropipets filled with $2 \mathrm{~m} \mathrm{NaCl}$ having resistances of 1-3 M . Bipolar stimulating electrodes, made by twisting together 2 pieces of Formvar-insulated $62-\mu \mathrm{m}$-diameter tungsten wire, were placed in stratum radiatum and/or stratum oriens to activate synaptic inputs to the pyramidal cells or in the alveus to discharge the cells antidromically. Unless stated otherwise, stimuli consisted of $0.1-\mathrm{msec}-$ duration current pulses at 10-150 mA. In general, stimulus intensities were adjusted for a consistent discharge of a small fraction of the target cells, evoking a 1-2 mV population spike (Andersen et al., 1971). Normal stimulation frequency was once per minute. High-frequency stimuli were delivered at $100 \mathrm{~Hz}$ and were the same intensity as those used to establish baseline responses. A computer was used to digitize, automatically measure, and continuously display changes in population spike amplitude. The slope of the positive field EPSP recorded from the CA1 cell layer was analyzed off-line at the conclusion of the experiment. Slope measures had to increase a minimum of $5 \%$, and population spike amplitudes $10 \%$, following high-frequency stimulation in order for a treatment to have been considered effective. Statistical comparisons were made using the Student's $t$ test.

For pharmacological studies, agents were dissolved in the medium at 100- to 1000-fold their final desired concentration and were then added to the perfusion solution to achieve the appropriate dilution using a syringe pump. Compounds employed included phencyclidine (PCP; final concentration, $10 \mu \mathrm{M}$ ) and 2-amino-5-phosphonovaleric acid (AP5; final concentrations, 20 and $50 \mu \mathrm{M}$ ).

\section{In vivo preparation}

Animals and surgery. Male Sprague-Dawley albino rats weighing 280$350 \mathrm{gm}$ at the time of surgery were obtained from Harlan Laboratories. The animals were group housed (2-4/cage) with a $12 \mathrm{hr}$ light/dark cycle and were given food and water ad libitum. All rats were water deprived for approximately $16 \mathrm{hr}$ prior to surgery. They then received injections of atropine methyl nitrate $(0.2 \mathrm{mg} / \mathrm{kg}$, i.p.). One hour later the animals were anesthetized with secobarbital $(50 \mathrm{mg} / \mathrm{kg}$, i.p.); supplemental doses (5-8 $\mathrm{mg}$ ) were given at approximately $1 \mathrm{hr}$ intervals.

After surgical anesthesia had been established, as judged by the loss of both corneal blink reflex and overt response to tail pinch, the rat's head was placed in a stereotaxic frame. The hair overlying the skull was clipped and a longitudinal incision made. The skull was cleared of connective tissue and leveled, and holes were drilled for support screws, ground and stimulator indifferent sites, and a microdrive base. The electrical ground consisted of a pair of $250-\mu \mathrm{m}$-diameter, Teflon-covered stainless steel wires from which the insulation had been removed from the last $3 \mathrm{~mm}$ from the tips. The uninsulated portions of these wires were placed into the prefrontal cortex bilaterally. (Coordinates, from bregma, were AP $+2.0 \mathrm{~mm}$, ML $1.0 \mathrm{~mm}$.) The stimulator indifferent electrode, a $125 \mu \mathrm{m}$ stainless steel wire insulated except at the tip, was placed in the left cortex (AP $-6.0 \mathrm{~mm}$, ML $2.0 \mathrm{~mm}$ ).

The recording site was located over the right dorsal hippocampus (AP $-4.2 \mathrm{~mm}$, ML $2.5 \mathrm{~mm})$. The stimulating electrode $(125 \mu \mathrm{m}$ stainless steel wire insulated except at the tip) was aimed at the ventral hippocampal commissure on the left side (AP $-1.8 \mathrm{~mm}$, ML $1.0 \mathrm{~mm}$ ). The dura was reflected over each site. A microdrive (Deadwyler et al., 1979) fitted with a tungsten microelectrode (impedance $0.7-1.0 \mathrm{M} \Omega$ at $1 \mathrm{kHz}$ ) was positioned over the recording site, and the electrode was lowered into the CAl pyramidal cell layer. The stimulating electrode was then lowered into the commissural pathway. Final stimulator placement was determined by optimizing the amplitude of the population spike recorded in response to commissural stimulation $(1-10 \mathrm{~V}, 160 \mathrm{msec} \mathrm{du}-$ ration pulses). Following this procedure, the recording electrode and microdrive were removed, leaving the microdrive base; the base, as well as the stimulating, indifferent, and ground electrodes were then cemented in place using dental acrylic. Connecting leads from the electrodes were gathered into a socket made using a Cannon ITT Centi-loc plastic strip. This socket was also cemented to the skull.

Experimental procedure. Animals were allowed at least $7 \mathrm{~d}$ to recover from surgery. Recordings were never made from a rat until its preoperative body weight had been reestablished. All recording was done in a Plexiglas enclosure $(21 \times 22 \mathrm{~cm})$ placed in a sound-attenuating chamber. On the day before testing, a microdrive loaded with a tungsten electrode was reattached to the base cemented on the animal's skull. A wire from the recording electrode was plugged into the socket mounted on the rat's head. The animal was placed in the recording chamber, and a cable into which an FET-operational amplifier $(10 \times$ gain) had been built was then also plugged into the head socket. The amplified signal from the electrode was led through a slip-ring commutator (Micco, 1977) to a second-stage amplifier, which further boosted the signal gain to a total of $100 \times$. Neuronal activity was filtered at broad $(0.5-7500 \mathrm{~Hz})$ and narrow (500-7500 $\mathrm{Hz}$ ) bandwidth to monitor evoked potentials and unit activity simultaneously. The recording electrode was lowered into the CAl pyramidal cell layer, after which it was raised approximately $400 \mu \mathrm{m}$. The animal remained in the recording chamber for approximately $8 \mathrm{hr}$; during this period it was fed either raisins or a mixture of rat chow and sugar.

The following day, the recording electrode was again lowered into the pyramidal cell layer. Following a $2-4 \mathrm{hr}$ wait, stimulation of the commissural pathway was begun. Stimuli were delivered every $30 \mathrm{sec}$, at an intensity that reliably evoked a population spike which was approximately $25 \%$ of the maximum response. Baseline stimulation was not begun until motor activity had ceased and the rat was either in slow wave sleep or in a quiet awake state. Monitoring of the hippocampal EEG allowed for the distinction to be made between slow wave sleep and REM sleep. Following 5-10 min of stimulation, the animal was awakened by opening the door of the recording chamber. Within 5-15 sec of waking, a high-frequency stimulus train (described below) was delivered. This procedure facilitated presentation of the stimulus train to all the rats while they were in a relatively stable and reproducible behavioral state; all animals exhibited a stereotyped posture composed of standing motionless and looking out at the experimenter following opening of the chamber door. Within 20-60 sec after the high-frequency train, the animals were again resting quietly on the floor of the recording chamber; at this point, single stimulus pulses were again presented. Data were acquired only during the resting, awake, or slow wave sleep behavioral conditions; data obtained during movement or REM sleep were discarded. Recordings continued for at least $10 \mathrm{~min}$ following the highfrequency train in all cases. If PB potentiation occurred, the electrode was raised and the microdrive removed at the conclusion of the session. If no change in the response was seen, the stimulation protocol was repeated 2-3 d later. Recordings were made several times (3-8) from the same rat over the course of 1-6 months in order to test the reproducibility of the effects. At the conclusion of the last experiment, the animal was overdosed with secobarbital and perfused with formalin, and the brain sectioned coronally to confirm recording and stimulating electrode placements. 


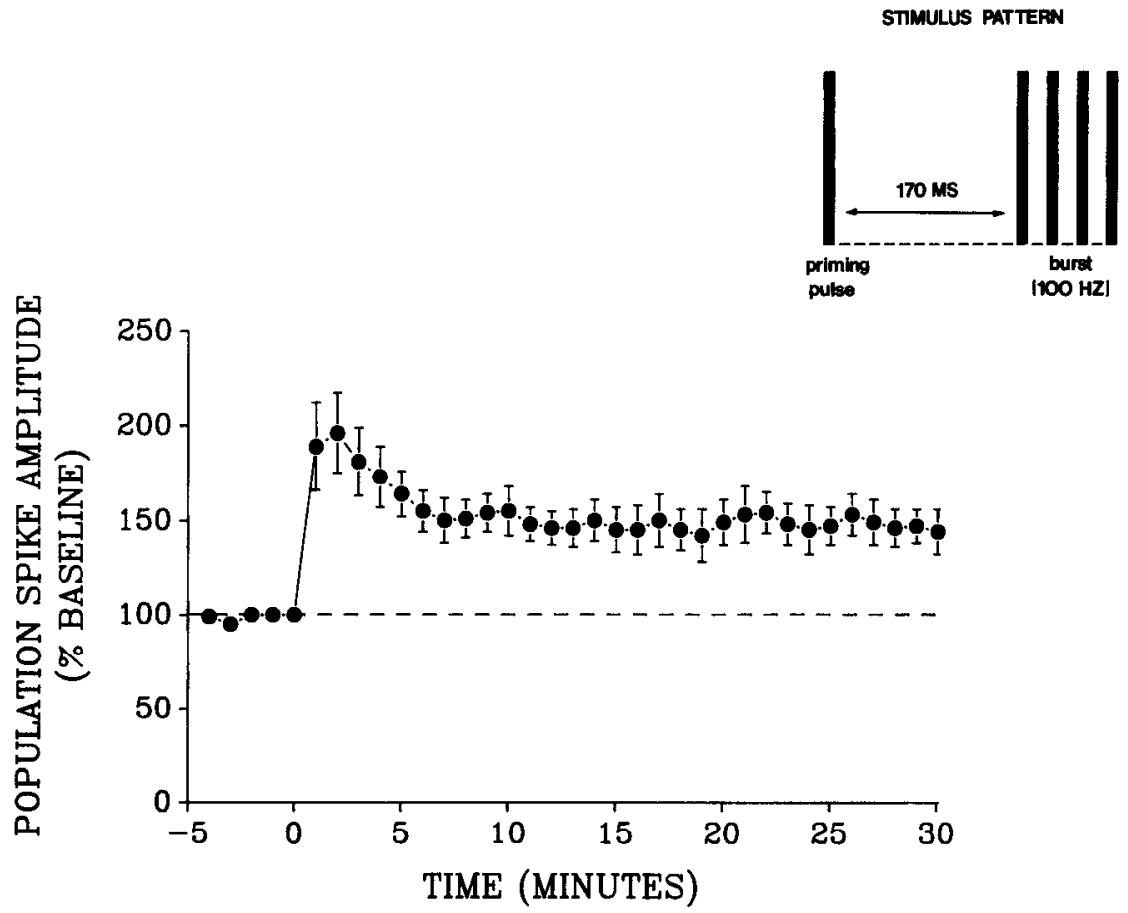

Figure 1. Primed burst potentiation in vitro. Presentation of a patterned train of 5 stimuli $(1+4$; see inset at upper right) to the Schaffer/commissural afferents in stratum radiatum resulted in a long-lasting increase in population spike amplitude recorded in the CA1 pyramidal cell layer. Each point represents the mean \pm SEM $(n=6)$. In this and all similar figures, stimulation trains were delivered at time 0 .
Stimulation trains were of 3 types: (1) 5 pulses at $200 \mathrm{~Hz}$; (2) a PB train consisting of a single pulse followed $170 \mathrm{msec}$ later by a burst of 4 stimuli at $200 \mathrm{~Hz}$; and (3) a conventional train of stimuli known to elicit LTP (e.g., 100 pulses at $100 \mathrm{~Hz}$ or a pair of 4 pulse trains separated by $170 \mathrm{msec}$, presented 5 times in $10 \mathrm{sec}$, for a total of 40 stimuli). The frequency of burst stimulation was increased from 100 to $200 \mathrm{~Hz}$ in the in vivo experiments in order to more closely mimic the interval timing of spike activity we have observed most often in hippocampal spike activity, i.e., $5 \mathrm{msec}$, corresponding to $200 \mathrm{~Hz}$. All stimuli within a single session, i.e., both single pulses and high-frequency trains, were delivered at the same voltage and duration. High-frequency stimulation was given between 1300 and $1500 \mathrm{hr}$ in all cases.

Analysis of the slope of the EPSP and amplitude of the population spike, and statistical analyses were performed in the same manner described earlier for the in vitro experiments.

\section{Results}

In vitro effects

As we have previously reported (Rose and Dunwiddie, 1986), a patterned train of 5 pulses delivered via a single Schaffer/ commissural stimulating electrode (a single priming pulse followed $170 \mathrm{msec}$ later by a burst consisting of 4 pulses at 100 $\mathrm{Hz}$ ) induced a long-lasting elevation in the magnitude of the CA1 population spike evoked by subsequent single stimuli. An increase was seen in 46 of 55 attempts (84\%); for these cases, the change recorded 10 min after PB train delivery was $153 \pm$ $10 \%$ (mean \pm SEM) of control $(p<0.01)$. Population spike amplitudes were maximally increased at $1 \mathrm{~min}$ after PB stimulation. The average response then declined until a plateau was reached after about $5 \mathrm{~min}$; from this point, essentially no further decrement was seen for observation periods of up to $30 \mathrm{~min}$ (Fig. 1). Growth in the slope of the positive field EPSP recorded in the pyramidal cell layer was seen less often ( 13 of 28 , or $46 \%$, of cases analyzed); for the experiments showing an increase, the slope was increased to $111 \pm 2 \%$ of control $(p<0.05)$. Other patterns of 5-pulse stimulation, e.g., 5 stimuli at $100 \mathrm{~Hz}$, or reversing the order of the priming pulse and the burst, did not produce lasting effects (see also Rose and Dunwiddie, 1986).
The window of effective interstimulus intervals between the priming pulse and the burst was found to be quite narrow (Fig. 2 , top). Of the periods tested, only intervals of 140 and 170 msec produced lasting increases in the population spike (146 \pm $18 \%$ and $153 \pm 10 \%$ of control, respectively; $p<0.01$ for both); intervals of $70 \mathrm{msec}$ or less, or of $500 \mathrm{msec}$ or greater, had no long-term effect. Using $170 \mathrm{msec}$ spacing, pairing the single priming pulse with as few as 2 burst stimuli induced a significant lasting increase in the population spike $(p<0.05)$. From this threshold, the magnitude of the change was linearly proportional to the number of burst stimuli presented (up to 10 tested; Fig. 2 , bottom). Presentation of 10 pulses at $100 \mathrm{~Hz}$, not preceded by a priming pulse, resulted in a lasting increase in population spike amplitude in only 1 of 7 attempts.

The priming pulse and burst stimuli used to induce PB potentiation did not have to be delivered to the same set of afferent fibers. Stimulation of separate populations of inputs to either the apical or basal dendritic fields of the pyramidal neurons (e.g., the priming pulse to stratum oriens, followed by the burst to stratum radiatum, or vice versa) appeared to be as effective in producing PB potentiation as when all the stimuli were administered via the same electrode. In these cases, only the burststimulated input was potentiated (Fig. 3, top).

The characteristics of heterosynaptic PB potentiation were further examined using a pair of stimulating electrodes placed in stratum radiatum to activate separate populations of Schaffer/ commissural inputs (Dunwiddie and Lynch, 1978). The magnitude of heterosynaptically induced PB potentiation was not significantly different from the homosynaptic effect $(173 \pm 19 \%$ vs $153 \pm 10 \%, p>0.1$; Fig. 3, bottom). Also using the heterosynaptic paradigm, PB potentiation was induced using a lowintensity priming pulse that did not evoke a population spike $(143 \pm 11 \%, p<0.05$; Fig. 3 , bottom). In contrast, antidromic activation of the pyramidal cells was not effective as a priming pulse; in this experiment, only a transient (approximately $5 \mathrm{~min}$ ) increase in the response was observed (Fig. 3, bottom). 

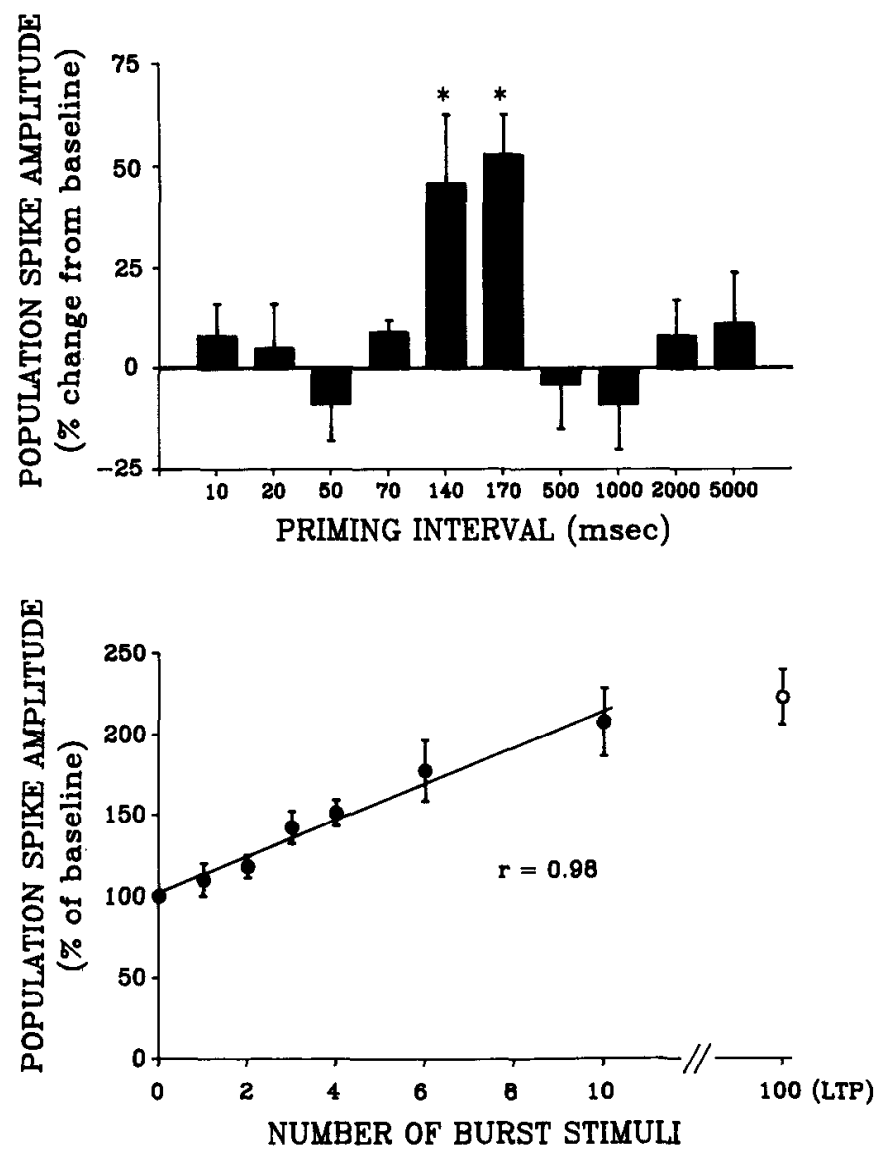

Figure 2. Top, Effect of varying the interval between the priming pulse and the burst on PB potentiation. Of the intervals tested, only 140 and $170 \mathrm{msec}$ (corresponding to frequencies of $6-7 \mathrm{~Hz}$ ) produced lasting changes in population spike amplitude. Bars represent means \pm SEM of at least 8 values for each time point, taken $10 \mathrm{~min}$ after the delivery of a $1+4$ stimulus train. ${ }^{*} p<0.01$. Bottom, Influence of the number of stimuli in the burst on PB potentiation. Using a single priming pulse and a 170 msec interval between the priming pulse and the burst, at least 2 burst stimuli were necessary to evoke a significant change from control $(p<0.05)$. Increasing the number of stimuli in the burst resulted in proportionally larger effects; this relationship was linear within the range of $1-10$ burst stimuli $(p<0.005)$. However, the increase observed following an LTP-inducing stimulus $(100 \mathrm{~Hz} / 1 \mathrm{sec})$ fell well below the regression line. The delivery of 10 stimuli at $100 \mathrm{~Hz}$ without the priming pulse did not produce lasting effects. Control ( 0 burst) stimuli were single pulses presented once per minute. Each point represents the mean \pm SEM of minutes $8-10$ following PB delivery; $n \geq 6$ for each group.

Under normal circumstances, delivery of the 4-pulse burst without a preceding priming pulse did not have any lasting effect on population spike amplitude (e.g., Fig. 3, bottom). However, if the duration of each stimulus pulse in the burst was increased 10 -fold, from 0.1 to $1 \mathrm{msec}$, an increase in the population spike could be evoked by 4 stimuli at $100 \mathrm{~Hz}$ without a preceding priming pulse $(132 \pm 12 \%$ of control after $10 \mathrm{~min} ; n=7 ; p<$ $0.05)$.

\section{Relationship of PB potentiation to LTP}

LTP was induced by the delivery of a single $100 \mathrm{~Hz}, 1 \mathrm{sec}$ stimulus train to the Schaffer/commissural afferents to the pyramidal cells. Separate slices from the same group of animals employed for studies of PB potentiation were used. This stimulation was effective in inducing a lasting enhancement in population spike amplitude in 32 of 35 attempts (91\%); the average
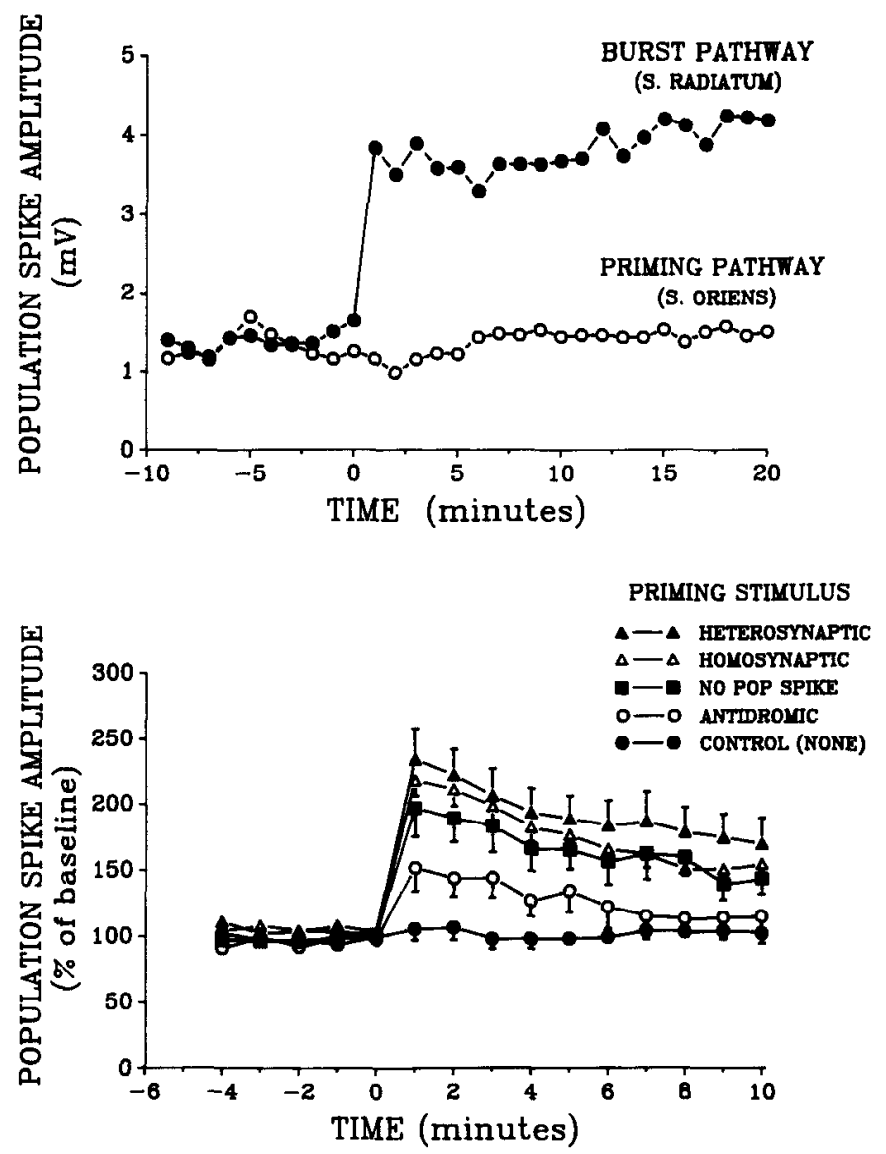

Figure 3. Top, Heterosynaptically induced PB potentiation. Stimulating electrodes were placed in stratum oriens (Priming pathway) and stratum radiatum (Burst pathway) to activate separate inputs to the pyramidal neurons. A priming pulse was administered (at time 0) to the stratum oriens pathway, followed $170 \mathrm{msec}$ later by a 4-pulse burst to the stratum radiatum afferents. Only the response to stratum radiatum stimulation was subsequently increased. Bottom, Effects of various types of priming stimuli on PB potentiation. A priming pulse was delivered to stratum radiatum afferents which were cither the same as (Homosynaptic, $n=40$ ) or different from (Heterosynaptic, $n=12$ ), those which received the burst (4 pulses at $100 \mathrm{~Hz}$ ); both evoked robust $\mathrm{PB}$ potentiation. A low-intensity priming pulse (No pop spike, $n=6$ ), tested using the heterosynaptic paradigm, was also effective. However, an antidromic priming stimulus (Antidromic, $n=7$ ) did not produce a lasting change. In the heterosynaptic paradigms, only the input that received the burst demonstrated an elevation in population spike amplitude. Control stimulation $(n=10)$ consisted of the 4-pulse burst alone.

increase, measured $10 \mathrm{~min}$ after high-frequency stimulation, was $223 \pm 17 \%(p<0.001)$. The slope of the positive field EPSP recorded in the pyramidal cell layer increased in 12 of 24 , or $50 \%$, of the cases analyzed; the slope increased to 115.0 $\pm 1.2 \%$ of control $(p<0.01)$.

The relationship of PB potentiation to LTP was explored using both physiological and pharmacological experiments. Two approaches were used to investigate for possible interactions between PB and LTP trains. As we have previously described (Rose and Dunwiddie, 1986), when several LTP-inducing (100 $\mathrm{Hz} / 1 \mathrm{sec}$ ) trains were delivered at $10 \mathrm{~min}$ intervals, the increment in population spike with each succeeding train became smaller until, after 3-5 trains, no further increase in the response was observed. (After each train, the stimulation voltage was reduced to restore the potentiated population spike to its original amplitude.) PB stimulation $(1+4)$ administered at this point 
elicited an increase in the population spike in only 1 of 9 cases; on average, no significant change was observed.

A second method of examining the interaction between PB potentiation and LTP involved comparing the increases in population spike amplitude evoked by the LTP-inducing stimulus delivered alone with the increase observed following prior administration of one or more PB trains. As is illustrated in Figure 4(top), on average, LTP induced after a single PB train was smaller than that seen without a preceding $P B$ train and was still smaller if $2 \mathrm{~PB}$-inducing trains were given before the LTP stimulus ( $p<0.05$ after 2 PB trains). Finally, 3 PB trains resulted in a saturation effect, in which no significant changes in the response occurred following subsequent LTP stimulation $(p>0.1)$.

Recent work has shown that the induction of LTP can be prevented by agents that are antagonists of the $N$-methyl-Daspartate (NMDA) excitatory amino acid receptor (Collingridge et al., 1983; Stringer and Guyenet, 1983; Harris et al., 1984). The effects of 2 such compounds, PCP and AP5, on PB potentiation were studied. PCP $(10 \mu \mathrm{M})$ or AP5 $(20$ and $50 \mu \mathrm{M})$ were introduced into the perfusion medium at least $10 \mathrm{~min}$ before any experimental manipulations were attempted. After this period, a PB train was delivered, followed $10 \mathrm{~min}$ later by an LTPinducing stimulus. AP5 at concentrations $>20 \mu \mathrm{M}$, but not 10 $\mu \mathrm{M}$ PCP, caused consistent reductions in the amplitude of the baseline population spike; in these cases, the stimulus voltage was increased to return the response to predrug amplitude. In the presence of $20 \mu \mathrm{M}$ AP5, PB potentiation was reduced by $75 \%(n=6)$, although LTP was less affected (mean reduction of $54 \% ; n=6$ ). However, $50 \mu \mathrm{M}$ AP5, as well as $10 \mu \mathrm{M} \mathrm{PCP,}$ caused essentially a complete block of both $\mathrm{PB}$ potentiation and LTP (Fig. 4, bottom).

\section{In vivo effects}

\section{Characteristics of the CAl evoked response in the awake} animal

The field responses recorded in the CA1 pyramidal cell layer following commissural stimulation were divisible into 2 classes, based on the characteristics of the EPSP preceding the population spike. Class I responses consisted of a well-developed positive EPSP which was abruptly interrupted by the population spike $(n=13)$; for class II responses, the positivity that preceded the population spike was poorly developed or completely lacking $(n=13)$. In addition to analyzing all CA1 population spikes as a single group, the 2 classes of responses were analyzed separately; since no statistically significant differences were observed, only the combined data are presented. However, since the initial EPSP component was not reliably present in class II responses, these recordings were not included in EPSP slope analyses.

\section{Effects of patterned stimulation}

Data were obtained from 9 subjects in 26 recording sessions. PB potentiation occurred in the majority of the recordings (17/ 26 , or $65 \%$ ). This effect was obtained in $78 \%(7 / 9)$ of the subjects. The effects of PB stimulation on the population spike amplitude were monitored for a minimum of $20 \mathrm{~min}$. An increase in the slope of the EPSP was induced in 54\% (7/13) of the cases analyzed (class I responses only).

An example of the effect of PB stimulation on both the population spike amplitude and the slope of the EPSP of a class I response is shown in Figure 5. Both response measures remained significantly above the baseline for the duration of the recording
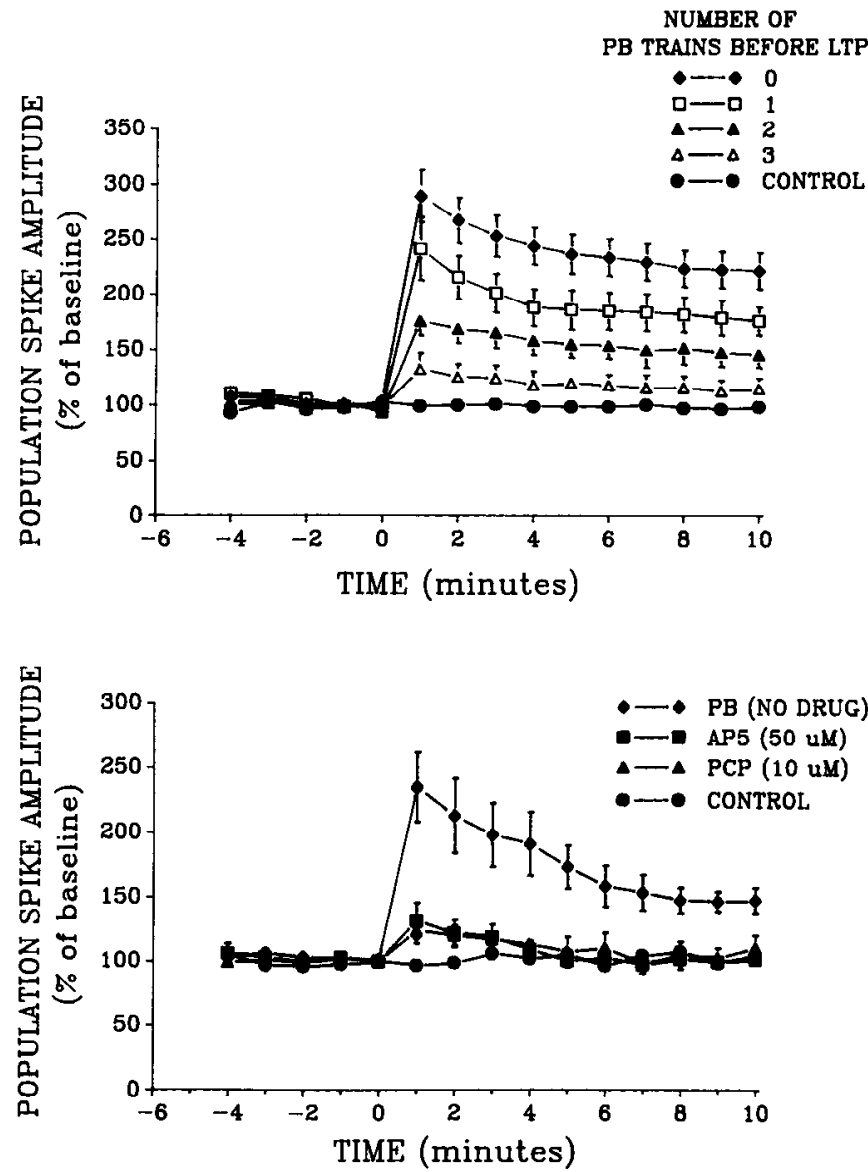

Figure 4. Top, Effect of PB potentiation on LTP. The delivery of 1 ( $n$ $=10), 2(n=9)$, or $3(n=3) \mathrm{PB}(1+4)$ trains prior to an LTP-inducing stimulation caused successive decrements in the enhancement of population spike amplitude normally evoked by the LTP $(100 \mathrm{~Hz} / 1 \mathrm{sec} ; n$ $=35$ ) train. Control stimuli were single pulses delivered once per minute $(n=11)$. Bottom, Effects of AP5 and PCP on PB potentiation. At the concentrations employed, both AP5 $(n=16)$ and $\operatorname{PCP}(n=4)$ prevented the increase in population spike amplitude normally seen following a PB $(1+4 ; n=28)$ stimulus train. Control stimuli were single pulses presented once per minute $(n=9)$.

session (45 min post-PB stimulation). Although most recordings were terminated 20-60 min after PB stimulation, in a small number of cases we continued to monitor the enhancement of response for up to $4 \mathrm{hr}$. Figure 6 illustrates one example of an increase in population spike amplitude that remained elevated for at least $3 \mathrm{hr}$ after PB stimulation.

The averaged data for the increases in population spike amplitude and EPSP slope are presented in Figure 7. Both response measures showed a large increase immediately following the PB train. From the initial peak values seen at $1 \mathrm{~min}$ following patterned stimulation, the responses declined somewhat until a plateau was reached at approximately $10 \mathrm{~min}$. At this point, the amplitude of the population spike was $170 \pm 17 \%$ (mean \pm SEM) of baseline, while the EPSP slope increased to $140 \pm 10 \%$ of control. Values obtained during the remainder of the recording session were not significantly different from this period.

In contrast to the $\mathrm{PB}$ effects, an unprimed stimulus train consisting of 5 stimuli at $200 \mathrm{~Hz}$ never had a lasting (>5 min) effect on either the population spike or the slope of the EPSP (0/17 attempts in 9 subjects; Fig. 7). In each of the 17 sessions in which unprimed stimulation was given, $\mathrm{PB}$ stimulation was 

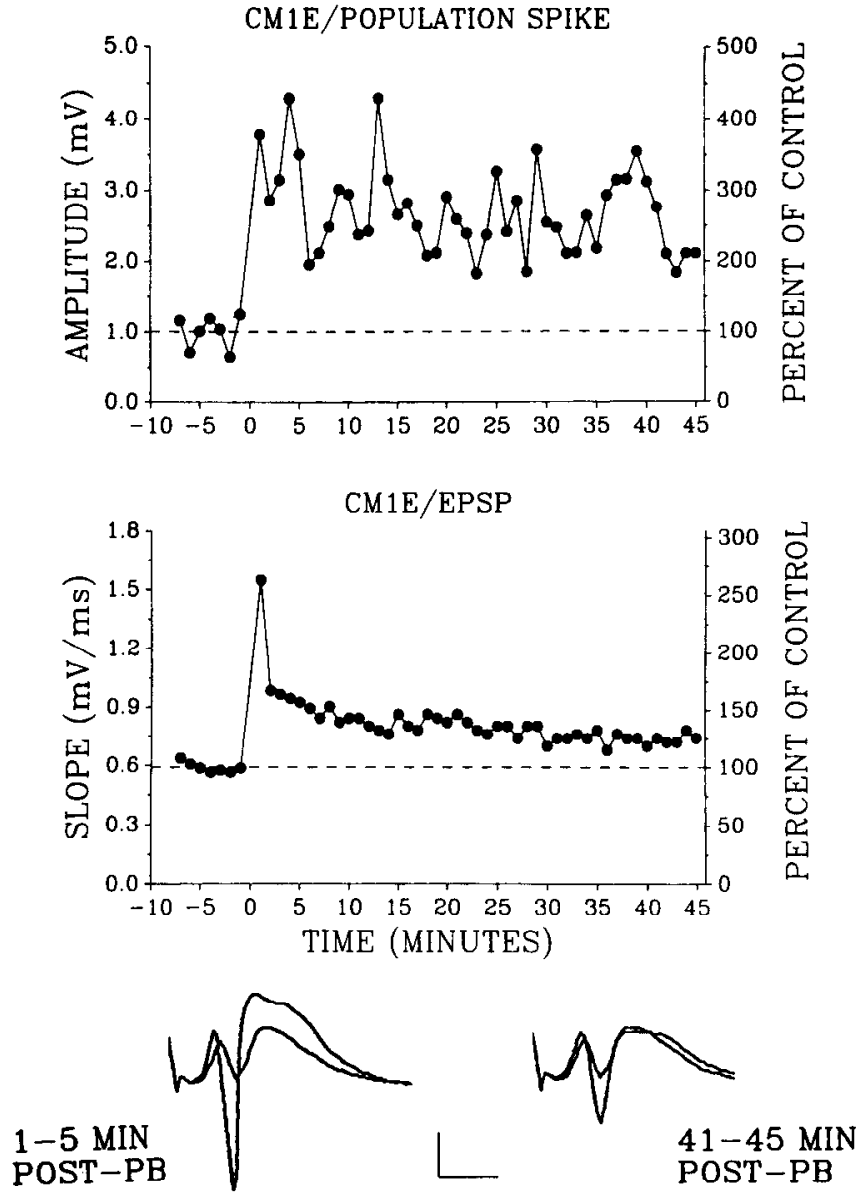

Figure 5. PB potentiation in the awake rat. Presentation of a patterned train of 5 stimuli (a single priming pulse followed $170 \mathrm{msec}$ later by a burst of 4 pulses at $200 \mathrm{~Hz}$ ) to the commissural afferents resulted in lasting increases in both the amplitude of the population spike (top) and the slope of the EPSP (middle) recorded in the CA1 pyramidal cell layer. The PB train was delivered at time 0 . The data for both measures are presented in terms of actual amplitude (left ordinate), as well as percentage change from the baseline response period (right ordinate). At the bottom are shown averaged responses obtained $1-5 \mathrm{~min}(l e f i)$ and 41-45 min (right) following PB stimulation. These records are superimposed upon the control average for purposes of comparison. Pre- and posttrain stimuli were delivered every $30 \mathrm{sec}$. In this and all subsequent figures, the 2 responses obtained in each minute were averaged to obtain $1 \mathrm{~min}$ mean values. The data were obtained from a single recording session (CM1E). Scale bars: $0.5 \mathrm{mV}, 5 \mathrm{msec}$.

administered 10-20 min later. In the majority of these cases (9/ $17,53 \%$ ), PB potentiation then occurred. This finding indicates that the ineffectiveness of unprimed stimulation was not a consequence of the system's inability to alter its response following high-frequency stimulation.

In the final experiment, we compared the magnitude of effects induced by conventional LTP stimulation and PB stimulation. The effects of LTP stimulation (40-100 pulses at high frequency) was significantly greater than that evoked by PB stimulation (359 $\pm 45 \%$ vs $170 \pm 17 \%$ of baseline $10 \mathrm{~min}$ after highfrequency stimulation). Although the LTP effect was approximately twice as large as PB potentiation, it was induced by 8 20 times the number of pulses.

\section{Discussion}

In a previous report, we showed that the threshold for the induction of lasting changes in the strength of hippocampal syn-

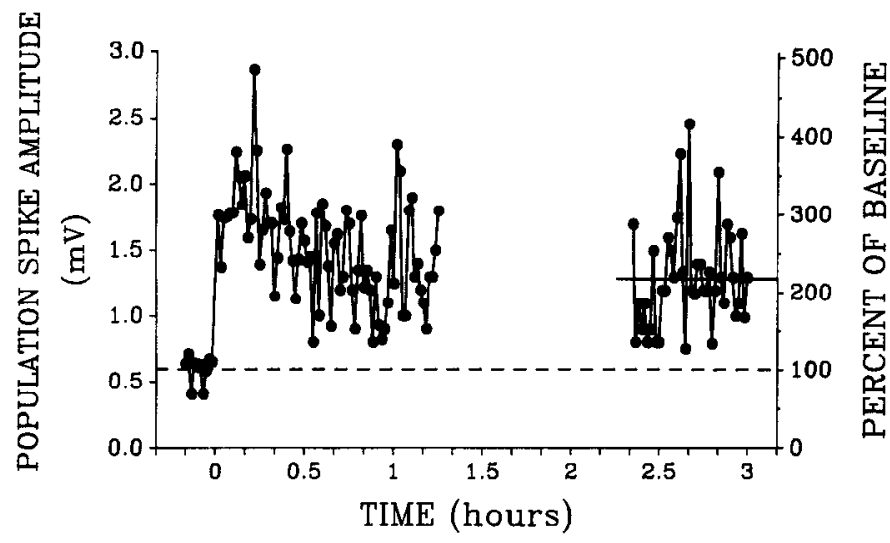

Figure 6. $\quad$ PB potentiation can last at least $3 \mathrm{hr}$. Following patterned stimulation (time 0), a significant increase in the amplitude of the population spike was seen. The response was monitored continually for approximately $1.25 \mathrm{hr}$. After this, a break in data acquisition took place, during which the animal was left undisturbed; test stimulation was resumed $1 \mathrm{hr}$ later. The solid line through the data points at the right indicates that the mean amplitude of the population spike was still elevated 2.4-3 hr after PB train delivery, the increase averaging $220 \%$ of bascline.

apses is markedly reduced when the stimulation is presented in an appropriate temporal pattern (Rose and Dunwiddie, 1986). Delivery of 5 pulses, separated into a single priming pulse followed 170 msec later by a high-frequency burst of 4 pulses, induced an increase in the amplitude of the in vitro CAl population spike. This effect was termed PB potentiation.

In the present study, we have provided a 2-tiered analysis of $\mathrm{PB}$ potentiation. The experiments performed on the in vitro hippocampal slice provide novel information concerning the effects of patterned stimulation on synaptic responses: (1) The threshold for PB potentiation is extremely low, requiring only 3 pulses to induce lasting changes; (2) the involvement of NMDA receptors is necessary for $\mathrm{PB}$ potentiation to occur; (3) following a saturation of $P B$ potentiation with multiple stimulation trains, long trains of "unpatterned" pulses do not induce a further increase, i.e., PB stimulation can saturate LTP effects; and (4) somal discharge in response to the priming pulse is neither a necessary nor sufficient condition for PB potentiation to occur. The last point is based on 2 complementary findings: An antidromic priming pulse is ineffective at inducing PB potentiation, and a priming pulse that is subthreshold for a population spike (indicating very little or no somal discharge activity) is effective at inducing $\mathrm{PB}$ potentiation. Thus, synaptic activation, not somal discharge, is critical for PB potentiation to occur. In a second level of analysis, we demonstrated that $\mathrm{PB}$ potentiation can be induced in the behaving animal.

\section{Comparative aspects of $\mathrm{PB}$ potentiation \\ Comparison of in vitro and in vivo effects}

The validity of the in vitro preparation is dependent on the assumption that intrinsic components of hippocampal function, including mechanisms of plasticity, remain intact once this structure is removed from the brain. Since PB potentiation has now been observed in both the intact hippocampus of behaving animals and the in vitro hippocampal slice, it is possible to compare the effects obtained in the 2 preparations.

Overall, the incidence and magnitude of the PB potentiation effects in the 2 preparations are remarkably similar. Three as- 
pects of PB potentiation effects obtained in vivo and in vitro were not significantly different: (1) the incidence of lasting changes in the population spike $(84 \%$ in vivo vs $65 \%$ of the in vitro recordings); (2) the incidence of changes in the slope of the positive EPSP recorded in the pyramidal cell layer (54\% in vivo vs $48 \%$ in vitro); and (3) the magnitude of $\mathrm{PB}$ potentiation of the population spike ( $170 \%$ of baseline in vivo vs $153 \%$ of baselinc in vitro). The only characteristic that differed between the 2 preparations was the magnitude of the slope of the EPSP $(140 \%$ of baseline in vivo vs $111 \%$ of baseline in vitro). The basis for the EPSP difference is not known, but it probably has its origins in differences between the intact hippocampus and the isolated hippocampal slice in terms of the degree of synaptic activation and routes of current flow. Nevertheless, the important point is that the $\mathrm{PB}$ potentiation effect is readily obtained in both the in vitro and in vivo hippocampus, and the incidence of the effects is similar. The findings indicate that the mechanisms underlying pattern-sensitive plasticity are present in the behaving animal and are largely unaffected by preparation of the tissue for in vitro recording procedures.

\section{Comparison of $P B$ potentiation to $L T P$}

We performed a comparison of PB potentiation to LTP using both physiological and pharmacological experiments. In the physiological analyses, one major finding is that the patterned stimulation of as few as 3 pulses ( 1 priming pulse followed 170 msec later by a burst of 2 pulses) can induce a lasting increase in population spike amplitude. This enhancement of response increased linearly with each additional burst pulse until 11 pulses $(1+10)$ evoked a response that was equivalent to the effect seen after a 1 -sec-long train of 100 pulses (Fig. 2, bottom). In the in vivo experiments, a continuous train of up to 20 times the number of pulses used in PB stimulation induced an LTP effect that was only twice as large as PB potentiation. Even 50 times as much stimulation of CA1 in the behaving animal (250 pulses; Buzsaki, 1980) induced an increase in response that was only twice that of the present study. These findings indicate a greater per pulse eflicacy for patterned stimulation versus a "nonpatterned" continuous train of pulses.

For both PB and LTP stimulation, increases in the population spike were more likely to occur than increases in the slope of the EPSP. A possible explanation for this result is that recording the field EPSP at the cell body layer does not provide an accurate reflection of the current flow into distant dendritic regions. However, it has also been observed by several investigators that lasting changes in population spike amplitude occur following high-frequency stimulation without accompanying changes in the EPSP (e.g., Bliss and Lømo, 1973; Andersen et al., 1980). Given this dissociation between the EPSP and population spike, it may be that pharmacological tests (see below) would provide a better means of distinguishing different forms of synaptic plasticity.

In the present study, the time course of $\mathrm{PB}$ potentiation and LTP was followed for at least $30 \mathrm{~min}$ in vitro (Fig. 1) and for up to $3 \mathrm{hr}$ in the awake rat (e.g., Fig. 6). In both preparations, the effects appeared to be highly stable. Although these results do not allow a direct comparison of the time course of the decay of $\mathrm{PB}$ potentiation and LTP, other studies provide data relevant to this issue. Buzsaki (1980) showed that extensive high-frequency stimulation ( $50 \mathrm{~Hz}$ for $5 \mathrm{sec}$ ) resulted in an increase in the strength of the commissural to $\mathrm{CA} 1$ pathway in behaving rats; the enhancement of response was maximal in the first $2 \mathrm{~d}$
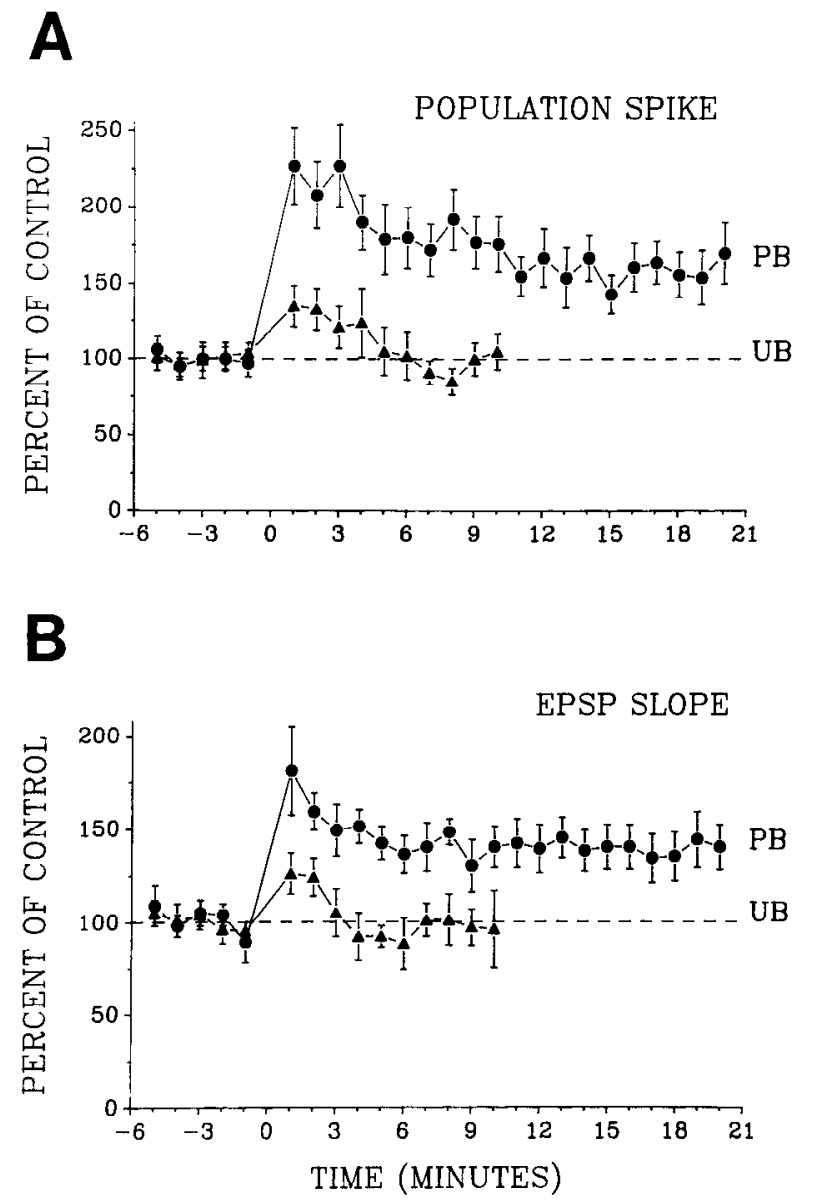

Figure 7. Summary data for $\mathrm{PB}$ and unprimed burst $(U B)$ potentiation in the awake rat. The PB train consisted of 5 patterned $(1+4)$ stimuli, while the UB train was 5 pulses at $200 \mathrm{~Hz}$. In all cases, stimulation was given at time $0 . A, \mathrm{~PB}$ stimulation induced a long-lasting increase in population spike amplitude $(n=17)$, while the UB stimulation evoked only a transient elevation in the response, which returned to baseline within $5 \mathrm{~min}(n=9)$. $B$, The slope of the positive EPSP recorded in the pyramidal cell layer was also enhanced following PB stimulation $(n$ $=7$ ), while the UB train evoked only a short-lasting increase $(n=5)$.

and then rapidly decayed toward baseline levels. In contrast, Staubli and Lynch (1987) demonstrated that stimulation of the commissural to CAl pathway resulted in an increase in synaptic efficacy that could last for at least 5 weeks. The major difference between the Buzsaki work and that of Staubli and Lynch is that the latter workers used patterned stimulation that was similar to the PB stimulation of the present study. Hence, in area CAl of the awake rat, patterned stimulation, as compared to continuous trains of pulses, appears to be a more effective means of inducing lasting changes in the strength of hippocampal synapses.

Evidence that similar mechanisms underlie LTP and PB potentiation was provided by a lack of additivity between the 2 types of stimulation. Specifically, if the 2 effects involve the same mechanisms, then a saturation of one process, e.g., PB potentiation, should prevent the effects of the other, e.g., LTP. We found that once asymptotic levels of PB potentiation were reached (after 3-5 PB trains), subsequent LTP stimulation had no lasting effects (Fig. 4, top). Similarly, multiple LTP trains eliminated the effects of PB stimulation. The lack of additivity 
of $P B$ potentiation and LTP suggests that the 2 types of responses share the same mechanism.

Our final comparison of PB potentiation and LTP demonstrated that the 2 procedures induce lasting changes using the same neurotransmitter/receptor complex. Recent work has demonstrated that the induction of LTP can be prevented by agents that are antagonists of the NMDA amino acid receptor, e.g., PCP and AP5 (Collingridge et al., 1983; Stringer and Guyenet, 1983; Harris et al., 1984). We have provided the first evidence that PCP and AP5 can block the induction of PB potentiation. Thus, the induction of $\mathrm{PB}$ potentiation appears to require the release of an excitatory amino acid, such as glutamate or aspartate, followed by activation of the NMDA receptor.

All of our findings indicate that the mechanisms of synaptic change induced by PB stimulation arc similar, if not identical, to those induced with LTP stimulation. PB potentiation appears to differ from LTP only in that the induction process is more efficient. Although the mechanisms underlying the pattern sensitivity of $\mathrm{PB}$ potentiation are not known, it is documented that, following priming, there is a prolongation of the EPSP during the subsequent burst stimulation (Larson and Lynch, 1986). The basis for this priming-induced increase in excitability may be a decreased efficacy of inhibitory input (Pacelli et al., 1987) during the 140-200 msec period following a priming stimulus. The priming pulse seems to have an effect equivalent to bath application of picrotoxin or intracellular current injection utilized in other studies (Gustafsson et al., 1987).

This comparison of LTP and PB potentiation emphasizes a changing perspective on electrically evoked synaptic strengthening. Prior to studies of patterned stimulation and plasticity, the induction of LTP was considered to involve 3 variables: (1) the frequency of stimulation; (2) the duration of the stimulus train; and (3) the intensity of the stimuli delivered. An increase in each of these variables (to a saturation point) results in an increase in the incidence and magnitude of synaptic plasticity. Consideration of these 3 factors alone would lead one to the conclusion that the probability of eliciting LTP is sensitive primarily to the time-averaged density of stimulation, i.e., the most favorable conditions for evoking LTP involve activating the greatest number of synapses in the shortest amount of time. However, the present findings demonstrate that the pattern of stimulation can also be a critical variable in the induction of synaptic plasticity, especially since it appears to markedly reduce the amount of stimulation necessary to induce lasting effects.

\section{PB potentiation: a model of associative memory?}

LTP is currently considered to be the most likely candidate for an information-storage mechanism (Teyler and Discenna, 1984, 1987). Although this proposal is largely accepted at a global level, the relationship of hippocampal LTP to specific aspects of memory formation is unknown. One pertinent aspect of PB potentiation is its critical dependence on the temporal components of stimulation. As this effect may be relevant to hippocampal-dependent learning, we now evaluate PB potentiation, and also conventional LTP, as models of associative memory.

The concept of "associative" LTP was first formulated by Levy and Steward (1979). These authors demonstrated that a lasting increase in responsiveness can be induced in a previously subthreshold pathway only if it is stimulated concurrently with a stronger, suprathreshold input. The connotation of "associativity" as applied to LTP refers to a literal relationship of the experimental parameters of electrical stimulation to behavioral associative learning (see Levy and Steward, 1983; Kelso and Brown, 1986). However, the parameters of associative LTP induction differ markedly from those of behavioral learning. In animals, as well as human subjects, associative learning occurs only if there is a minimum delay between the onset of a relatively neutral stimulus, e.g., light (conditioned stimulus, or CS) and the onset of an arousing, biologically significant stimulus, e.g., food (unconditioned stimulus, or US). In fact, if the US is presented less than $100 \mathrm{msec}$ after the onset of the CS, subjects show either very poor or no learning (Kimble and Reynolds, 1967; Hoehler and Thompson, 1980). Nevertheless, in associative LTP, optimal "pairing" of the weak input (analogous to the CS) with the strong input (analogous to the US) occurs when the 2 pathways are stimulated either synchronously (Levy and Steward, 1979; Barrionuevo and Brown, 1983; Robinson, 1986; Sastry et al., 1986; Gustafsson et al., 1987) or with a delay of less than $25 \mathrm{msec}$ (Levy and Steward, 1983; Gustafsson and Wigström, 1986). These parameters of CS and US presentation would not support behavioral learning.

In the present study, the delay of $170 \mathrm{msec}$ between the weak stimulus (priming pulse) and strong stimulus (burst of 4 pulses) does satisfy the temporal constraints of behavioral associative learning, i.e., a separation of $170 \mathrm{msec}$ between a CS and US would lead to behavioral learning. However, certain features of PB potentiation limit its utility as a general model of associative learning, at least within the temporal constraints of classical conditioning training. First, in heterosynaptic PB potentiation, the pairing of priming (CS) with burst (US) stimulation results in a change in response in the US pathway, with no change observed in the CS pathway. As a possible model of associative learning, this finding would be inconsistent with a basic tenet of associative learning: Following repeated pairings, behavioral changes occur in response to the CS, and not in response to the US. Second, PB potentiation does not occur at PB intervals greater than or equal to $500 \mathrm{msec}$. Yet, in behavioral studies, a $500 \mathrm{msec}$ delay is the optimal interval for associating a CS with a US (Kimble, 1961; Kimble and Reynolds, 1967). Thus, as with conventional LTP, the parameters of PB potentiation do not allow for a literal comparison of the timing of electrical stimulation to the presentation of CS in behavioral tasks.

\section{Hypotheses on the functional significance of $\mathrm{PB}$ potentiation}

One of the most striking aspects of the present study was the narrow window of time following the priming pulse during which the burst of pulses induced lasting changes. Of all the intervals tested, only 2 (170 and $140 \mathrm{msec}$, corresponding to 6 and $7 \mathrm{~Hz}$, respectively) were effective. The significance of this time window between the priming pulse and the burst may relate to a prominent feature of hippocampal physiology, theta rhythm, which is a 4-12 Hz oscillation of the hippocampal EEG (Vanderwolf, 1969; Bland, 1986) and neuronal discharge activity (Rose, 1983). It is notable that frequencies outside the range of theta rhythm, e.g., $\leq 2 \mathrm{~Hz}$ and $\geq 14 \mathrm{~Hz}$, were ineffective.

The similarity between the temporal components of $\mathrm{PB}$ potentiation and theta rhythm suggests that changes in synaptic strength occur during behaviors correlated with endogenously generated theta rhythm. In rats, theta activity is observed during exploratory behavior and rapid eye movement (REM) sleep. Perhaps theta rhythm serves to prime hippocampal neurons, such that coincident synaptic activation from a separate input 
(e.g., complex spikes) exceeds the threshold for LTP. Support for this possibility is derived from behavioral experiments of Sharp and colleagues. These workers demonstrated that lasting changes in hippocampal responses occur when rats are placed in a spatially complex environment (Sharp et al., 1985, 1987). This increased response may have resulted from the interaction of a "priming" of the hippocampus by theta activity and neural activity specifically involved in the encoding of spatial information.

An alternative hypothesis is that an endogenous form of PB potentiation may be involved in memory consolidation processes suggested to take place during theta-related (REM) sleep. There is extensive documentation indicating that REM sleep appears to be critical for learning to occur; following learning there is an increased incidence of REM sleep, and deprivation of REM sleep results in a disruption of learning (see Smith, 1985, for review).

The actual relationship of patterned stimulation to mechanisms of memory formation remains unknown. To test the hypothesis that $\mathrm{PB}$ potentiation exogenously activates mechanisms that normally participate in inducing behaviorally related synaptic plasticity, the relationship between behavioral states correlated with theta rhythm and the threshold for the strengthening of hippocampal synapses needs to be addressed. Initial studies indicate that behavioral state is a powerful influence on the synaptic plasticity. For example, the induction of LTP is suppressed during a non-theta phase of sleep (Jones Leonard et al., 1987). In addition, a PB potentiation-like effect is preferentially induced when patterned stimulation coincides with the positive phase of electrically evoked theta rhythm in the anesthetized rat (Pavlides et al., 1988). These findings indicate that the threshold for the induction of hippocampal synaptic plasticity is a dynamic characteristic, which is highly dependent on the behavioral state and hippocampal EEG. Subsequent experiments will determine whether PB potentiation can be used as a tool to provide insight into the way in which endogenous rhythms modulate memory formation.

\section{References}

Andersen, P., T. V. P. Bliss, and K. K. Skrede (1971) Unit analysis of hippocampal population spikes. Exp. Brain Res. 13: 208-221.

Andersen, P., S. H. Sundberg, O. Sveen, J. W. Swann, and H. Wigström (1980) Possible mechanisms for long-lasting potentiation of synaptic transmission in hippocampal slices from guinea-pigs. J. Physiol. (Lond.) 302: 463-482.

Barnes, C. A. (1979) Memory deficits associated with senescence: A neurophysiological and behavioral study in the rat. J. Comp. Physiol. Psychol. 93: 74-104.

Barnes, C. A., and B. L. McNaughton (1985) In Memory Systems of the Brain: Animal and Human Cognitive Processes, N. M. Weinberger, J. L. McGaugh, and G. Lynch, eds., pp. 49-61, Guilford, New York.

Barrionuevo, G., and T. H. Brown (1983) Associative long-term potentiation in hippocampal slices. Proc. Natl. Acad. Sci. USA 80:73477351.

Bland, B. H. (1986) The physiology and pharmacology of hippocampal formation theta rhythms. Prog. Neurobiol. 26: 1-54.

Bliss, T. V. P., and T. Lomo (1973) Long-lasting potentiation of synaptic transmission in the dentate area of the anesthetized rabbit following stimulation of the perforant path. J. Physiol. (I ond.) 232: 331356.

Buzsaki, G. (1980) Long-term potentiation of the commissural pathCA1 pyramidal cell synapse in the hippocampus of the freely moving rat. Neurosci. Lett. 19: 293-296.

Buzsaki, G., L. S. Leung, and C. H. Vanderwolf (1983) Cellular bases of hippocampal $\mathrm{EEG}$ in the behaving rat. Brain Kes. Kev. 6: 139171.
Byrne, J. H. (1987) Cellular analysis of associative learning. Physiol. Rev. 67: 329-439.

Collingridge, G. L., and T. V. P. Bliss (1987) NMDA receptors-their role in long-term potentiation. TINS 10: 288-293.

Collingridge, G. L., S. J. Kehl, and H. McLennan (1983) Excitatory amino acids in synaptic transmission in the Schaffer collateral-commissural pathway of the rat hippocampus. J. Physiol. (Lond.) 334: 33-46.

Deadwyler, S. A., J. Biela, G. Rose, M. West, and G. Lynch (1979) A microdrive for use with glass or metal microelectrodes in recording from freely moving rats. Electroencephalogr. Clin. Neurophysiol. 47: 752-754.

Dunwiddie, T., and G. Lynch (1978) Long-term potentiation and depression of synaptic responses in the rat hippocampus: Localization and frequency dependency. J. Physiol. (Lond.) 276: 353-367.

Farley, J., and D. L. Alkon (1985) Cellular mechanisms of learning, memory and information storage. Annu. Rev. Psychol. 36: 419-494.

Greenstein, Y. J., C. Pavlides, and J. Winson (1988) Long-term potentiation in the dentate gyrus is preferentially induced at a theta rhythm periodicity. Brain Res. 438: 331-334.

Gustafsson, B., and H. Wigström (1986) Hippocampal long-lasting potentiation produced by pairing single volleys and brief conditioning tetani evoked in separate afferents. J. Neurosci. 6: 1575-1582.

Gustafsson, B., H. Wigström, W. C. Abraham, and Y.-Y. Huang (1987) Long-term potentiation in the hippocampus using depolarizing current pulses as the conditioning stimulus to single volley synaptic potentials. J. Neurosci. 7: 774-780.

Harris, E. W., A. H. Ganong, and C. W. Cotman (1984) Long-term potentiation in the hippocampus involves activation of N-methyl-Daspartate receptors. Brain Res. 323: 132-137.

Hoehler, F. K., and R. F. Thompson (1980) Effect of the interstimulus (CS-USC) interval on hippocampal unit activity during classical conditioning of the nictitating membrane response of the rabbit. J. Comp. Physiol. Psychol. 94: 201-215.

Jaffard, R., and Y. Jeantet (1981) Posttraining changes in excitability of the commissural path-CA1 pyramidal cell synapse in the hippocampus of mice. Brain Res. 220: 167-172.

Jones Leonard, B., B. L. McNaughton, and C. A. Barnes (1987) Suppression of hippocampal synaptic plasticity during slow wave sleep. Brain Res. 425: 174-177.

Kelso, S. R., and T. H. Brown (1986) Differential conditioning of associative synaptic enhancement in hippocampal brain slices. Science 232: 85-87

Kimble, G. A. (1961) Hilgard and Marquis' Conditioning and Learning, pp. 155-160, Appleton-Century-Crofts, New York.

Kimble, G. A., and B. Reynolds (1967) Eyelid conditioning as a function of the interval between conditioned and unconditioned stimuli. In Foundations of Conditioning and Learning, G. Kimble, ed., pp. 279-287, Appleton-Century-Crofts, New York.

Larson, J., and G. Lynch (1986) Induction of synaptic potentiation in hippocampus by patterned stimulation involves two events. Science 232: 985-988.

Larson, J., D. Wong, and G. Lynch (1986) Patterned stimulation at the theta frequency is optimal for the induction of hippocampal longterm potentiation. Brain Res. 368: 347-350.

Levy, W. B., and O. Steward (1979) Synapses as associative memory elements in the hippocampal formation. Brain Res. 175: 233-245.

Levy, W. B., and O. Steward (1983) Temporal contiguity requirements for long-term associative potentiation/depression in the hippocampus. Neuroscience 8: 791-797.

Lynch, G., and M. Baudry (1984) The biochemistry of memory: A new and specific hypothesis. Science 224: 1057-1063.

McNaughton, B. L. (1983) Activity dependent modulation of hippocampal synaptic efficacy: Some implications for memory processes. In The Neurobiology of the Hippocampus, W. Seifert, ed., pp. 233251, Academic, London.

Micco, D. J., Jr. (1977) Lightweight, multi-contact, slip-ring commutator for recording and stimulation with small animals. Brain Res. Bull. 2: 499-502.

Pacelli, G. J., F. Naghdi, and S. R. Kelso (1987) Possible role of inhibition in LTP induction. Soc. Neurosci. Abstr. 13: 977.

Pavlides, C., Y. J. Greenstein, M. Grudman, and J. Winson (1988) Long-term potentiation in the dentate gyrus is induced preferentially on the positive phase of theta rhythm. Brain Res. 439: 383-387.

Ranck, J. B., Jr. (1973) Studies on single neurons in dorsal hippocam- 
pal formation and septum in unrestrained rats. Part I. Behavioral correlates and firing repertoires. Exp. Neurol. 41: 462-531.

Robinson, G. B. (1986) Enhanced long term potentiation induced in rat dentate gyrus by coactivation of septal and entorhinal inputs: Temporal constraints. Brain Res. 379: 56-62.

Rose, G. (1983) Physiological and behavioral characteristics of dentate granule cells. In Neurobiology of the Hippocampus, W. Seifert, ed., pp. 449-472, Academic, London.

Rose, G. M., and T. V. Dunwiddie (1986) Induction of hippocampal long-term potentiation using physiologically patterned stimulation. Neurosci. Lett. 69: 244-248.

Sastry, B. R., J. W. Goh, and A. Auyeung (1986) Associative induction of posttetanic and long-term potentiation in CA1 neurons of rat hippocampus. Science 232: 988-990.

Sharp, P. E. B. L. McNaughton, and C. A. Barnes (1985) Enhancement of hippocampal field potentials in rats exposed to a novel, complex environment. Brain Res. 339: 361-365.

Sharp, P. E., C. A. Barnes, and B. L. McNaughton (1987) Effects of aging on environmental modulation of hippocampal evoked responses. Behav. Neurosci. 101: 170-178.

Smith, C. (1985) Sleep states and learning: A review of the animal literature. Neurosci. Biobehav. Rev, 9: 157-168.

Spencer, H. J., V. Gribkoff, C. Cotman, and G. Lynch (1976) GDEE antagonism of iontophoretic amino acid excitations in the intact hippocampus and in the in vitro hippocampal slice preparation. Brain Res. 105: 478-481.
Staubli, U., and G. Lynch (1987) Stable hippocampal long-term potentiation elicited by 'theta' pattern stimulation. Brain Res. 435: 227234.

Stringer, J. L., and P. G. Guyenet (1983) Elimination of long term potentiation in the hippocampus by phencyclidine and ketamine. Brain Res. 258: 159-164.

Suzuki, S. S., and G. K. Smith (1985) Burst characteristics of hippocampal complex spike cells in the awake rat. Exp. Neurol. 89: 9095.

Teyler, T. J., and P. Discenna (1984) Long-term potentiation as a candidate mnemonic device. Brain Res. Rev. 7: 15-28.

Teyler, T. J., and P. Discenna (1987) Long-term potentiation. Annu. Rev. Neurosci. 10: 131-161.

Vanderwolf, C. H. (1969) Hippocampal electrical activity and voluntary movement in the rat. Electroencephalogr. Clin. Neurophysiol. 26: 407-418.

Weisz, D. J., G. A. Clark, and R. F. Thompson (1984) Increased responsivity of dentate granule cells during nictitating membrane response conditioning in rabbit. Behav. Brain Res. 12: 145-154.

Wigström, H., and B. Gustafsson (1985a) Facilitation of hippocampal long-lasting potentiation by GABA antagonists. Acta Physiol. Scand. 125: $159-172$

Wigström, H., and B. Gustafsson (1985b) On long-lasting potentiation in the hippocampus: A proposed mechanism for its dependence on coincident pre- and postsynaptic activity. Acta Physiol. Scand. 123: 519-522. 